\title{
Studies on the Formation and Aggregation of Polyelectrolyte Complexes Formed between Poly(styrenesulfonic acid) and Poly(diallyl- $N, N$-dimethylammonium chloride)
}

\author{
T. SCHINDLER and E. NORDMEIER \\ University of Osnabrück, Department of Physical Chemistry, \\ Barbarastr. 7, D-49069 Osnabrück
}

(Received December 28, 1993)

\begin{abstract}
Special properties of polyelectrolyte complexes (PEC) formed between poly(diallyl- $N, N$-dimethylammonium chloride) (PDADMAC) and poly(styrenesulfonic acid) (PSSA) were studied by gravimetry, viscosimetry, potentiometry, and quasielastic light scattering. These complexes are unsoluble precipitates. Their stoichiometry is $1: 1$. Complex aggregation takes place only if a critical amount of salt is added. This critical salt concentration depends on the PEC concentration and the valence of the microions used. The aggregation behavior of the complexes was examined by quasielastic light scattering, varying the salt concentration. The data were analyzed by the theory of v. Smoluchowski. The accord between theory and experiment is qualitatively sufficient.
\end{abstract}

KEY WORDS Polyelectrolyte Complex / Aggregation / Poly(styrenesulfonic acid) / Poly(diallyl- $N, N$-dimethylammonium chloride) / Critical Salt Concentration /

It is well known that oppositely charged polyions can form unsoluble intermacromolecular complexes, so-called polyelectrolyte complexes (PEC). Thereby, cooperative interactions are very important. The stability of the complex depends on several parameters, such as polyion composition and chain length, concentration of microions, solvent, and temperature. Therefore it is very complicated to predict theoretically what happens, if two complementary polyions are mixed together. There only exist some qualitative or semiquantitative models for the interpolymer complex formation, but there is still no consistent theory for the description of reactions between polyelectrolytes.

The aggregation behavior of PECs plays an important role in many biological processes, such as the function of biomembranes, the self-assembly of proteins, and the transmission of genetic information. Thus, it is very interesting to investigate aggregation phenomena.
As there is little known about complexes of poly(styrenesulfonic acid) (PSSA)/poly(diallyl- $N, N$-dimethylammonium chloride) (PDADMAC), ${ }^{1}$ we have chosen this system for our investigations. The aim was threefold: first, we analyzed the complex stoichiometry and the degree of conversion of its formation. Second, we determined the complex sizes. Thereby, we varied the complex concentration, microion concentration, and microion valence. Third, the aggregation was examined. This was done by Quasielastic Light scattering (QELS). The data were analysed by the theory of $v$. Smoluchowski.

\section{EXPERIMENTAL}

\section{Materials and Preparation}

Sodium poly(styrenesulfonate) (NaPSS) $\left(M_{w}=5 \times 10^{5} \mathrm{~g} \mathrm{~mol}^{-1}\right)$ and PDADMAC $\left(M_{w}\right.$ $=2.4 \times 10^{5} \mathrm{~g} \mathrm{~mol}^{-1}$ ) were products of Polyscience Inc. The conversion of NaPSS 
to PSSA was done by ion-exchange using a Lewatit S100 column followed by dialysis against bidestilled water. The concentration of the stock solution was determined by titration. PDADMAC was used without further purification.

For the complex formation the aqueous solutions of PSSA and PDADMAC were mixed under stirring and stored under Argon for two days to reach equilibrium. This holds not for the solutions used for quasielastic light scatterning. In order to minimize the time for reaching temperature equilibrium with the index matching bath of the light scattering instrument, the solutions were centrifuged and tempered at $25^{\circ} \mathrm{C}$. Note, the samples were mixed immediately before measurement. The enthalpy of mixing is very small in any case. So it does not affect the temperature of the mixture.

\section{METHODS}

\section{Conductometry}

A Philips Model PW 9501 was used for Conductometric Measurements. The changes in conductivity after removal of the precipitate by centrifugation were negligible.

\section{Gravimetry}

The polyion solutions $\left(c=2.2 \times 10^{-2} \mathrm{M}\right)$ were mixed in a number of mole ratios. Note, all concentrations are in moles of monomer per liter. The mixtures were centrifuged at 13.000 $\mathrm{U} \mathrm{min}^{-1}$ for 30 minutes. The precipitate was washed several times with bidestilled water. Finally, it was dried under vacuum at $70^{\circ} \mathrm{C}$ and weighted. A change in the order of mixing does not affect the results.

\section{Quasielastic Light Scattering}

Principle. The theory of QELS has been thoroughly reviewed. Nevertheless, some principal features should be recalled. In our experiments we use the homodyne mode, that is, we measure the photocurrent autocorrela- tion function, $C(t)$. This can be written as

$$
C(t)=A+B|g(t)|^{2},
$$

where $|g(t)|$ is the normalized autocorrelation function of the scattered field and A and B are apparatus constants. For noninteracting identical particles, $|g(t)|$ is a single exponential. That is

$$
|g(t)|=\mathrm{e}^{-\Gamma t}
$$

$\Gamma$ is called the decay rate. It is related to the apparent translational diffusion coefficient $D_{\text {app }}$ by

$$
\Gamma=D_{\text {app }} \cdot q^{2},
$$

where $q$ is the wave vector. Extrapolation of $D_{\text {app }}\left(q^{2}\right)$ to $q^{2}=0$ gives the true diffusion coefficient, i.e., the diffusion coefficient of the center of gravity. It is designated $D$. According to the Stokes-Einstein relation

$$
D=\frac{\mathrm{k}_{\mathrm{B}} T}{6 \pi \eta_{0} R_{\mathrm{h}}}
$$

$D$ is related with the hydrodynamic radius $R_{\mathrm{h}}$. $\eta_{0}$ is the dynamic viscosity of the solvent. However, in most cases we have samples with a distribution of particle sizes. Then, $|g(t)|$ is a distribution of exponentials:

$$
|g(t)|=\int_{0}^{\infty} G(\Gamma) \mathrm{e}^{-\Gamma t} \mathrm{~d} \Gamma,
$$

where $G(\Gamma)$ is the normalized distribution of decay rates.

Equation 5 can be analyzed easily by the method of moments or cumulats. It consists in expanding $|g(t)|$ in a power series of $t$ :

$$
\begin{aligned}
& \ln |g(t)| \\
& \quad=-\bar{\Gamma} t+\frac{\mu_{2}}{2 ! \Gamma^{2}}(\bar{\Gamma} t)^{2}-\frac{\mu_{3}}{3 ! \overline{\Gamma^{3}}}(\bar{\Gamma} t)^{3}+\cdots
\end{aligned}
$$

where

$$
\bar{\Gamma}=\int_{0}^{\infty} \Gamma G(\Gamma) \mathrm{d} \Gamma
$$




$$
\mu_{i}=\int_{0}^{\infty}(\Gamma-\bar{\Gamma})^{i} G(\Gamma) \mathrm{d} \Gamma
$$

$\bar{D}$ is derived from $\bar{\Gamma}$ as $\bar{D}=\lim _{q^{2} \rightarrow 0} \bar{\Gamma} / q^{2}$, where $\bar{D}$ is the $z$-average diffusion coefficient.

Apparatus. An Argon-ion laser (wavelength $488 \mathrm{~nm}$; Spectra Physics) was used as light source. All experiments were performed at $25^{\circ} \mathrm{C} \pm 0.1^{\circ} \mathrm{C}$. An interference filter, calibrated for the wavelength of the incident beam, was placed in front of the photomultiplier. The output signal was analyzed on a 1024-channel ALV-3000 correlator. Further details are given in ref 4 .

\section{RESULTS AND DISCUSSION}

\section{Yield and Stoichiometry}

Figure 1 shows a plot of the precipitation yield $Y$ related to the amount of PDADMAC versus the monomer molar ratio, $N_{\mathrm{c}}$, of PSSA to PDADMAC. For $N_{\mathrm{c}}=n_{\text {PSSA }} / n_{\text {PDADMAC }} \in$ $[0,1], Y$ increases linearly from 0 to 1 . In this range, the yield related to the amount of PSSA is 1 . For $N_{\mathrm{c}} \geq 1, Y$ is 1 . Consequently, the degree of conversion is quantitative and no soluble complex is formed. ${ }^{3}$ That is, PDADMAC reacts like a pendant-type polycation. The linear increase of $Y$ for $N_{\mathrm{c}} \leq 1$ and its constancy for $N_{\mathrm{c}} \geq 1$ indicate a complex stoichiometry of $1: 1$.

To verify the gravimetric data we used the method of conductometric titration. This procedure is described in ref 5. In water, the degree of dissociation of PSSA is only 0.42 . Nevertheless, PSSA is a strong polyelectrolyte. The remaining protons are bound to the polyion chain due to strong electrostatic forces. $^{2}$ When the complex formation takes place, the polyion concentration decreases and the concentration of microions in the mixture increases because all counterions are released. The equivalent conductance of the microions is about three times higher than that of the polyions removed. Thus, the total conductivity of the solution, $\kappa$, increases.

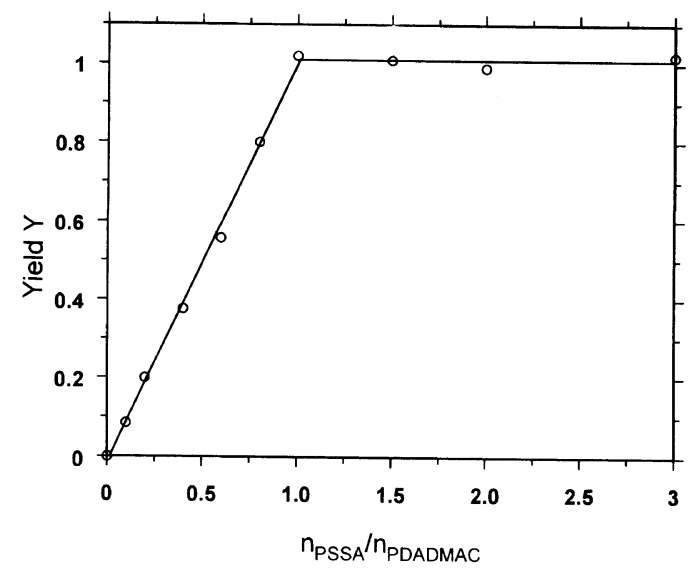

Figure 1. Yield $Y$ related to the amount of PDADMAC versus the molar ratio of PSSA to PDADMAC.

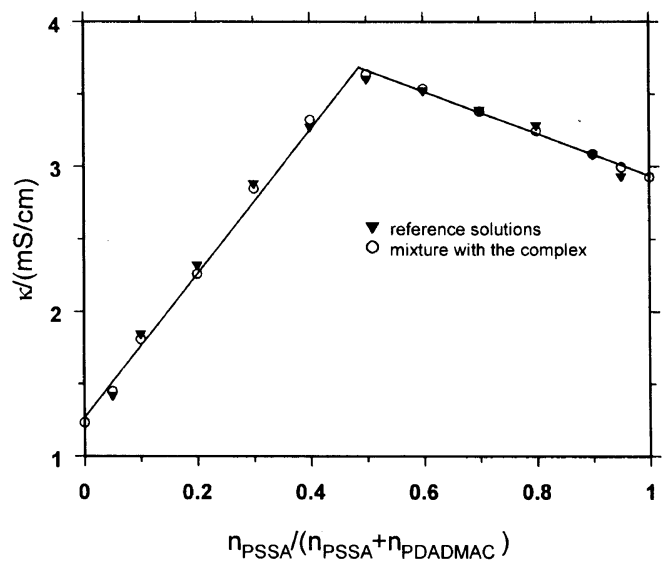

Figure 2. Conductances of the complex mixtures and the control mixtures versus the molar fraction of PSSA.

We compared the conductometric results with data obtained from reference solutions. These reference solutions were prepared as follows. For instance, mixing $5 \mathrm{ml}$ of $2.2 \times$ $10^{-2} \mathrm{M}$ PSSA with $15 \mathrm{ml}$ of $2.2 \times 10^{-2} \mathrm{M}$ PDADMAC, $\kappa$ should be equal to the value of a solution containing $1.1 \times 10^{-2} \mathrm{M}$ PDADMAC and $5.5 \times 10^{-3} \mathrm{M} \mathrm{HCl}$, if the degree of conversion is 1 . Thus, we measured the conductivity of the samples and their corresponding reference solutions.

Figure 2 shows a plot of the conductivity, $\kappa$, versus the monomer molar fraction $n_{\mathrm{PSSA}} /$ $\left[n_{\text {PSSA }}+n_{\text {PDADMAC }}\right]$ of PSSA. The circles present 
the data obtained for the complexes and the triangles are due to the data of the reference solutions.

The agreement between both data sets is very close. This supports the evidence that the complex formation is nearly quantitative. First, the conductivity increases linearly. The maximum is reached at the molar fraction 0.5 , which corresponds to the molar ratio $1: 1$. At this point all polyion counterions are released.

A further increase of the molar ratio results in a conductivity decrease. This is due to the fact that the counterion release of free PSSA is smaller than the release of reacted PSSA. In addition, the ion mobility of the macromolecules is lower. The linearity of $\kappa$ indicates that the complex composition is equimolar in any case.

\section{Complex Size}

The hydrodynamic radii, $R_{\mathrm{h}}$, were measured by QELS. The concentration, $c_{\text {complex }}$, was varied in the range of $5 \times 10^{-5} \mathrm{M}$ to $2.2 \times$ $10^{-3} \mathrm{M}$.

The results are shown in Figure 3. The sedimentation of the complexes formed is negligible over the time of the measurement.

$R_{\mathrm{h}}$ increases linearly with $c_{\text {complex }}$. This can be explained as follows. The number of polyion contacts is small at low $c_{\text {complex }}$ and large at high $c_{\text {complex }}$. Contacts between oppositely charged polyions lead to complexes. The higher $c_{\text {complex }}$, the larger is the probability for complex formation between more than one pair of polyions. Consequently, $R_{\mathrm{h}}$ increases as $c_{\text {complex }}$ is raised. Note, over a time of 10 days no further aggregation of the particles could be measured.

The extrapolation $\lim _{\mathrm{c} \rightarrow 0} R_{\mathrm{h}}$ gives the hydrodynamic radius $R_{\mathrm{h}, \min }=33.8 \mathrm{~nm}$. This radius is small in comparison to the radius $R_{\mathrm{h}}=150 \mathrm{~nm}$ of a single PSSA polyion $\left(M_{w}=\right.$ $500,000 \mathrm{~g} \mathrm{~mol}^{-1}$ ) when no salt is present. The relatively small value of $R_{\mathrm{h}, \min }$ points to a strong contraction of the polyions within the complex, caused by hydrophobic interactions.

There are two further parameters influencing

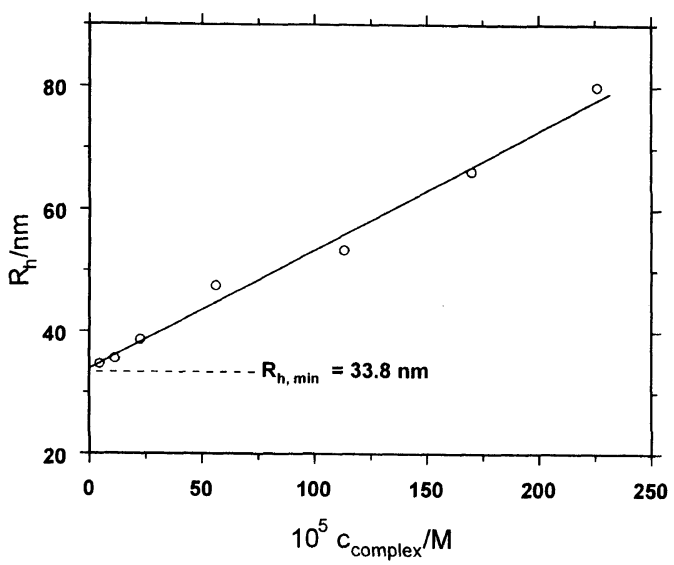

Figure 3. Hydrodynamic radius, $R_{\mathrm{h}}$, versus the complex concentration.

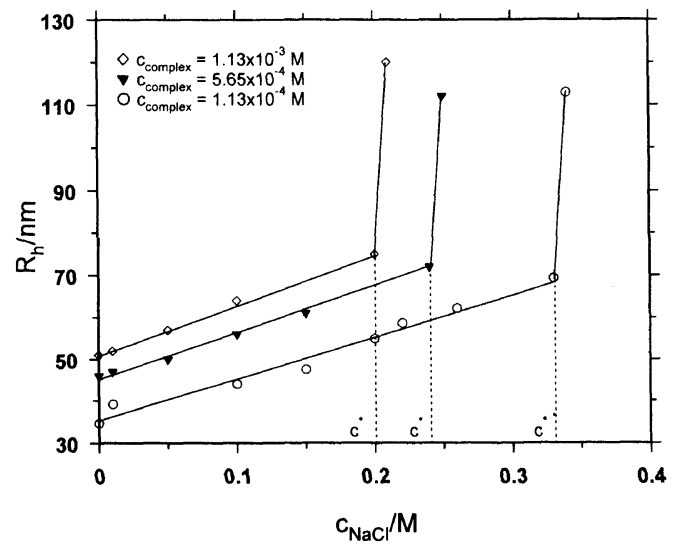

Figure 4. Hydrodynamic radius of the complexes versus the $\mathrm{NaCl}$ concentration. The measurements were performed one minute after mixing.

complex formation: salt concentration and the type of the microions added to the polyion solutions. We used equimolar polyion mixtures. The measurements were performed 1 minute after mixing the polyion solutions. Figure 4 shows the effect on $R_{\mathrm{h}}$ when $\mathrm{NaCl}$ is added. Three different polyion concentrations are considered. With increasing salt concentration the hydrodynamic radius increases linearly until a critical $\mathrm{NaCl}$ concentration, $c_{\mathrm{NaCl}}^{*}$, is reached. At this point, the complexes start to aggregate. $c_{\mathrm{NaCl}}^{*}$ decreases with increasing complex concentration, $c_{\text {complex }}$ A similar observation was made by Tsuchida. ${ }^{6,7} \mathrm{He}$ 


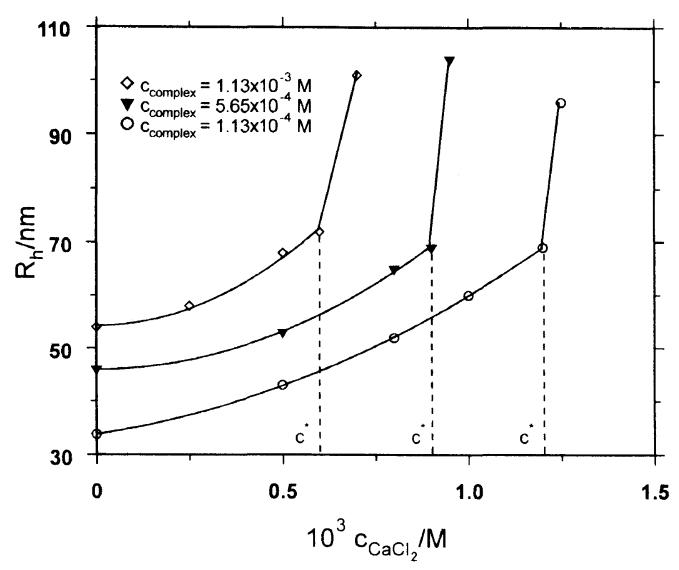

Figure 5. Hydrodynamic radius of the complexes versus the $\mathrm{CaCl}_{2}$ concentration. The measurements were performed one minute after mixing.

Table I. Summary of critical salt concentrations

\begin{tabular}{|c|c|c|c|c|}
\hline$\frac{c_{\text {complex }}}{\mathrm{M}}$ & Salt & $\frac{c^{*}}{\mathrm{M}}$ & $\frac{c_{\mathrm{NaCl}}^{*}}{c_{\mathrm{CaCl}_{2}}^{*}}$ & $\frac{R_{\mathrm{h}}^{*}}{\mathrm{~nm}}$ \\
\hline $\begin{array}{l}1.13 \times 10^{-4} \\
1.13 \times 10^{-4}\end{array}$ & $\begin{array}{l}\mathrm{NaCl} \\
\mathrm{CaCl}_{2}\end{array}$ & $\begin{array}{c}0.33 \\
1.2 \times 10^{-3}\end{array}$ & 275 & $\begin{array}{l}69 \\
68\end{array}$ \\
\hline $\begin{array}{l}5.65 \times 10^{-4} \\
5.65 \times 10^{-4}\end{array}$ & $\begin{array}{l}\mathrm{NaCl} \\
\mathrm{CaCl}_{2}\end{array}$ & $\begin{array}{c}0.24 \\
9.0 \times 10^{-4}\end{array}$ & 267 & $\begin{array}{l}71 \\
68\end{array}$ \\
\hline $\begin{array}{l}1.13 \times 10^{-3} \\
1.13 \times 10^{-3}\end{array}$ & $\begin{array}{l}\mathrm{NaCl} \\
\mathrm{CaCl}_{2}\end{array}$ & $\begin{array}{c}0.20 \\
6.0 \times 10^{-4}\end{array}$ & 333 & $\begin{array}{l}75 \\
72\end{array}$ \\
\hline Error & & & $10 \%$ & $5 \%$ \\
\hline
\end{tabular}

found that for complexes based on hydrogen bonds a critical $\mathrm{pH}$ exists. Above this critical $\mathrm{pH}$ the complexes do not aggregate over a time scale of tens of minutes.

Next, we considered the effect of the valence of the microions added. In Figure $5 R_{\mathrm{h}}$ is plotted versus the concentration of $\mathrm{CaCl}_{2}$. Again we used 3 concentrations. The same trend is found as in Figure 4. However, now for $c_{\mathrm{CaCl}_{2}}<c_{\mathrm{CaCl}_{2}}^{*}$ the hydrodynamic radius increases according to a second order law $\left(R_{\mathrm{h}} \sim c_{\mathrm{CaCl}_{2}}^{2}\right)$ and $c_{\mathrm{CaCl}_{2}}^{*}$ is about a factor of 100 lower than $c_{\mathrm{NaCl}}^{*}$.

Table I gives a summary of the results abstracted from Figures 4 and 5. Column 4 presents the ratios of the critical concentration
$c_{\mathrm{CaCl}}^{*}$ to the critical concentration $c_{\mathrm{CaCl}_{2}}^{*}$ for a given complex concentration. It is seen that $c_{\mathrm{NaCl}}^{*} / c_{\mathrm{CaCl}_{2}}^{*}$ is nearly constant over the range of complex concentrations studied. The critical hydrodynamic radii $R_{\mathrm{h}}^{*}$ are summarized in column 5 . They are also constant. In addition, it seems that $R_{\mathrm{h}}^{*}$ is independent of the valence of the microions used. The average value of $R_{\mathrm{h}}^{*}$ is $70 \mathrm{~nm}$. This seems to be the critical size of a complex for aggregation process to start.

\section{THE AGGREGATION AND ITS KINETIC ANALYSIS}

\section{Time Dependency of $R_{\mathrm{h}}$}

A quite interesting field of research is the kinetic of complex aggregation. Our handling was as follows. $c_{\mathrm{NaCl}}$ and $c_{\text {complex }}$ were fixed and $R_{\mathrm{h}}$ was observed as a function of time, $t$. Three series of measurements were performed. All measurements were done at $25^{\circ} \mathrm{C}$; the complex concentration was $1.13 \times 10^{-4} \mathrm{M}$. The salt concentrations used were chosen in the order of $c_{\mathrm{NaCl}}^{*}$. We prepared samples with $c_{\mathrm{NaCl}}=0.34$ $\mathrm{M}, 0.38 \mathrm{M}$, and $0.40 \mathrm{M}$.

Unfortunately, the time range is limited by the smallest and largest detectible sizes of $R_{\mathrm{h}}$. If $t$ is small, the experimental error is high because the aggregation continues during the measurement. So we determined only the mean value of the measurement. If $t$ is large, the complexes are too large. Then, they are no longer Rayleigh scatterers.

Figure 6 shows the results. $R_{\mathrm{h}}$ increases continuarly with $t$. The data can be described well by the power law

$$
R_{\mathrm{h}}=R_{0} \times t^{b}
$$

Here, $R_{0}$ and $b$ are constants. Values of $R_{0}$, $b$, and the correlation coefficient, $s$, are listed in Table II. When $b<1$, the size increase-rate of the particles decreases with time. For $b>1$, the size increase-rate increases with time. A similar relationship was found by Hemker and Frank $^{8}$ for complexes based on hydrogen bonds. 


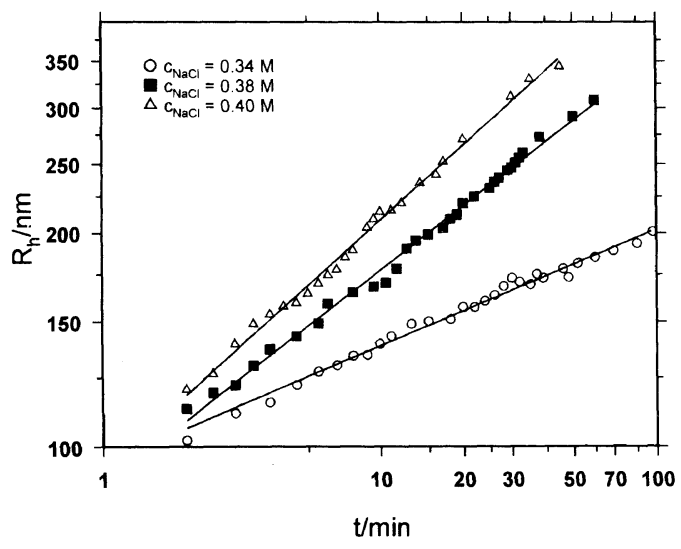

Figure 6. Hydrodynamic radius versus time for three different salt concentrations.

Table II. Aggregation parameters belonging to eq 1

\begin{tabular}{cccc}
\hline$\frac{c_{\mathrm{NaCl}}}{\mathrm{M}}$ & \multicolumn{1}{c}{$r_{0}$} & $b$ & $s$ \\
\cline { 2 - 4 } & $\mathrm{nm}$ & & \\
\hline 0.34 & 94.77 & 0.1647 & 0.9942 \\
0.38 & 86.45 & 0.3096 & 0.9977 \\
0.40 & 92.53 & 0.3521 & 0.9974 \\
\hline
\end{tabular}

\section{Size Distribution of the Complexes}

Another very interesting characteristic quantity of a complex is the complex size distribution. The inverse Laplace transformation of the photocurrent autocorrelation function gives the distribution of the translational diffusion coefficient, $D$. The relationship between molar mass, $M$, and $D$ is:

$$
M=\left(\frac{D}{K}\right)^{1 / \gamma}
$$

To calculate the molar mass distribution the constants $K$ and $\gamma$ have to be determined.

Studies of interpolymer complexes using electron microscopy have shown that the structure of the complexes is spherical during the primary phase of aggregation. Later they grow slowly to fibrous structures. ${ }^{6}$

Therefore, we assume that the PSSA/PDADMAC complexes are rigid spheres. The density of these spheres, $\rho$, is

$$
\rho=\frac{3 M}{4 \pi R_{\mathrm{h}}^{3}}
$$

Comparing eq 4 and 11 it can be seen that the molar mass, $M$, is proportional to $R_{\mathrm{h}}^{3}$, $D \sim M^{-1 / 3}$; that is $\gamma=1 / 3$. For the calculation of $K$, it is sufficient to know one value of $D$ and one of $M$. Two further assumptions are necessary. First, it is assumed that the complexes are monodisperse at the start phase of the aggregation. For a complex consisting of one PSSA molecule with $M_{w}=5 \times 10^{5} \mathrm{~g} \mathrm{~mol}^{-1}$, and every functional group of PSSA being bound to a functional group of PDADMAC, the molar mass, $M_{1}$, of a single complex is $8.4 \times 10^{5} \mathrm{~g} \mathrm{~mol}^{-1}$. The second assumption is that the value of $R_{\mathrm{h}}=33.8 \mathrm{~nm}$ extrapolated for infinite dilution (see Figure 3) belongs to a single pair of oppositely charged polyions. Inserting $R_{\mathrm{h}}=33.8 \mathrm{~nm}$ into eq 4 yields the translational diffusion coefficient $D=7.26 \times$ $10^{-12} \mathrm{~m}^{2} \mathrm{~s}^{-1}$. With eq 10 this gives $K=6.85 \times$ $10^{-10} \mathrm{~mol} \mathrm{~m}^{2} \mathrm{~s}^{-1} \mathrm{~g}^{-1}$.

Next, we transform the distribution function of the translational diffusion coefficient, $h_{\mathrm{D}}(D)$, into the molar mass distribution function, $h_{\mathrm{M}}(M)$.

The inverse Laplace transformation was performed by the program "Odil", developed by ALV Lasertechnik, Germany.

The distribution of the degree of aggregation, $h_{i}$ versus $i$ as obtained by QELS is defined as

$$
h_{i}=\frac{\sum m_{i}}{m_{\mathrm{tot}}}=\frac{N_{i} \cdot i \cdot m_{1}}{m_{\mathrm{tot}}},
$$

where $i$ is the number of single complexes in an aggregate, i.e., the degree of aggregation. $N_{i}$ is the number of aggregates of mass $m_{i}=i \cdot m_{1}$, and $m_{1}$ is the mass of one single complex at the beginning. $m_{\text {tot }}$ is the total mass of all particles. It is independent of time; that is, $m_{\text {tot }}=N_{0} \cdot m_{1}$, where $N_{0}$ is the number of complexes at the beginning (at time $t=0$ ). For our calculations we set $m_{1}=M_{1}$. 
To explain our experimental data we compared them with the aggregation theory developed by v. Smoluchowski. ${ }^{9}$ He describes the distribution of the degree, $i$, of aggregation as a function of time $t$. V. Smoluchowski supposes that the aggregates are rigid spherical particles, all having the same size at $t=0$. The aggregates formed during the aggregation are also assumed spherically. Further, no long range forces exist and every particle collision results in an aggregation. According to the second diffusion law, v. Smoluchowski derived the following equation:

$$
N_{i}=N_{0} \frac{\left(a N_{0} t\right)^{i-1}}{\left(1+a N_{0} t\right)^{i+1}}
$$

where

$$
a=\frac{2 \mathrm{k}_{\mathrm{B}} T}{3 \eta_{0}}=3.0821 \times 10^{-18} \mathrm{~m}^{3} \mathrm{~s}^{-1}
$$

Taking into account the condition $m_{i}=i \cdot m_{1}$ and inserting eq 13 into eq 12 we get

$$
h_{i}=i \times \frac{\left(a N_{0} t\right)^{i-1}}{\left(1+a N_{0} t\right)^{i+1}}
$$

At this point, it is necessary to compute the number of particles $N_{0}$ at $t=0$. The complex concentration, $c_{\text {complex }}$, is $1.13 \times 10^{-4} \mathrm{M}$. With a degree of polymerisation, $P$, of $2400, N_{0}$ is calculated as follows:

$$
N_{0}=\mathrm{N}_{\mathrm{A}} \times P \times c_{\text {complex }}=2.83 \times 10^{19} \mathrm{~m}^{-3}
$$

Then, the theoretical value of the product $a \times N_{0}$ is $87.4 \mathrm{~s}^{-1}$. To compare the theoretical data with the experimental one, both datasets are normalized as

$$
\int_{i=1}^{\infty} h_{i} d_{i}=1
$$

Figures 7 and 8 show the plots for the times 1, 5, and 10 minutes. The best agreement between theory and experiment is given if the theoretical curves are calculated with $a \times N_{0}=$ $0.171 \mathrm{~s}^{-1}$. This value is 500 times lower than the theoretical value of $87.4 \mathrm{~s}^{-1}$. The observed

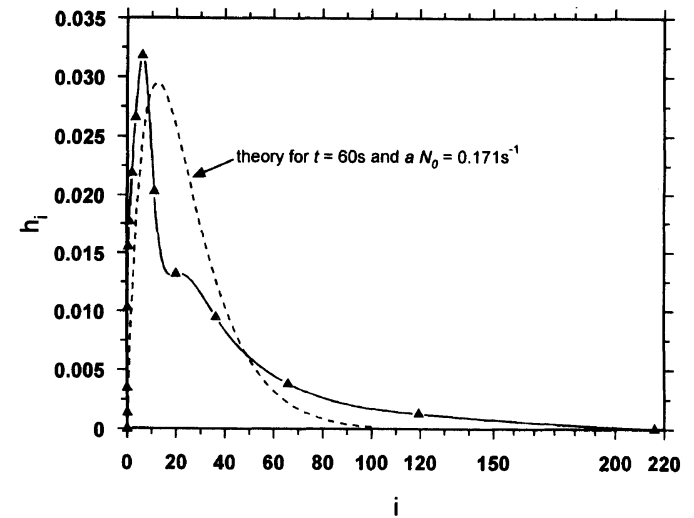

Figure 7. Experimental particle size distribution curve; comparison between theory and experiment.

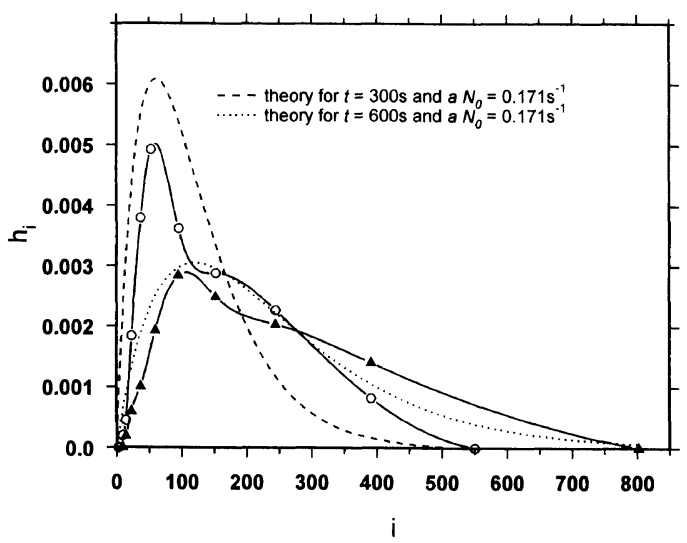

Figure 8. Experimental particle size distribution curves; comparison between theory and experiment.

aggregation process is much slower than predicted by theory. Repulsive forces between the complexes seem to be the reason for slowing the aggregation. The existence of a critical salt concentration also points to repulsive forces. These forces have to be compensated by increasing the microion concentration in the solution. Thus, $a \times N_{0}$ should be modified by a repulsive term. Further, it is seen that the experimental curves are broader than the theoretical curves. This may be due to the fact that the polyelectrolytes used are not monodisperse. Another interesting effect is that the experimental curves show two maxima. That is, the aggregates seem to form a bimodal molar 
mass distribution.

To analyse the time dependence of $a \times N_{0}$, we compared the theoretical behaviour of the average hydrodynamic radius with time to the experimental data. Therefore it is necessary to derive a relationship that describes the hydrodynamic radius as a function of the theoretical distribution of the degree of aggregation. The weight average molar mass is defined as

$$
M_{w}=\int_{0}^{\infty} h_{\mathrm{M}} M \mathrm{~d} M
$$

Inserting eq 15 into eq 18 and using $M_{i}=i \times M_{1}$ we obtain

$$
M_{w}(t)=M_{1} \int_{i=1}^{\infty} i^{2} \frac{\left(a N_{0} t\right)^{i-1}}{\left(1+a N_{0} t\right)^{i+1}} \mathrm{~d} i
$$

For $R_{\mathrm{h}}$ and $M_{w}$ we use the relation

$$
R_{\mathrm{h}}=\Phi \times M_{w}^{\gamma}
$$

For spherical particles $\gamma$ is $1 / 3$. Using the same assumptions as for the calculation of $K$, we find $\Phi$ is $3.58 \times 10^{-10} \mathrm{~m} \mathrm{~g}^{1 / 3} \mathrm{~mol}^{-1 / 3}$.

Inserting eq 20 into eq 19 we get

$$
R_{\mathrm{h}}(t)=\Phi \times\left[M_{1} \int_{i=1}^{\infty} i^{2} \frac{\left(a N_{0} t\right)^{i-1}}{\left(1+a N_{0} t\right)^{i+1}} \mathrm{~d} i\right]^{1 / 3}
$$

Equation 21 can be numerically integrated. The results are shown in Figure 9. They are compared to the experimental data. The observations are as follows. The increase of the theoretical curve is significantly larger than the increase of the experimental curve. Thus, it seems that the term $a \times N_{0}$ is not a constant. It may depend on time. The larger aggregates may tend to decay with time until an equilibrium is reached. Unfortunately, this equilibrium lies beyond the detection limit of our instrument.

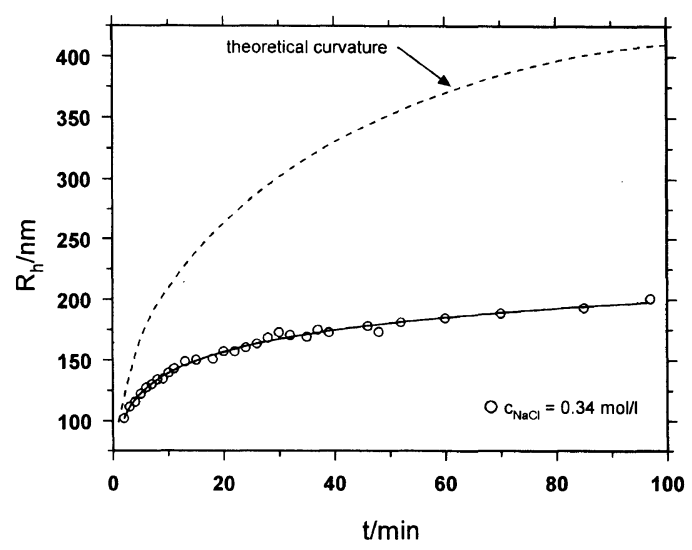

Figure 9. Hydrodynamic radius of the complexes compared to theoretical predictions.

Summarizing our work on aggregation, v. Smoluchowkis theory describes the aggregation of complexes qualitatively well. Nevertheless the aggregation process seems to be influenced by complex-complex interactions, so that these effects should be considered in the theory to get a quantiatively satisfactory relation between experimental and theoretical data.

\section{REFERENCES}

1. Y. Kurokawa, N. Shirakawa, M. Terada, and N. Yui, J. Appl. Polym. Sci., 25, 1645 (1980).

2. L. Kotin and N. Nagasawa, J. Am. Chem. Soc., 83, 1026 (1961).

3. V. A. Kabanov and A. B. Zezin, Pure \& Appl. Chem., 56, 343 (1984).

4. E. Nordmeier and M. D. Lechner, Polym. J., 21, 623 (1989).

5. A. S. Michaels, L. Mir, and N. S. Schneider, J. Phys. Chem., 69, 1447 (1965).

6. E. Tsuchida, Y. Osada, and H. Ohno, J. Macromol. Sci. Phys., B17, 683 (1980).

7. E. Tsuchida, H. Matsuda, and H. Ohno, Makromol. Chem., 182, 2267 (1981).

8. D. J. Hemker and C. W. Frank, Macromolecules, 23, 4404 (1990).

9. M. v. Smoluchowski, Z. Phys. Chem., 92, 129 (1917). 